\title{
ORIGINAL ARTICLE Benign Paroxysmal Positional Vertigo (BPPV) in Adult Patients between 15-40 years of age: A study on Treatment Outcome in Teaching Hospitals
}

\author{
ZAFAR IQBAL ${ }^{1}$, REHAN SALEEM ${ }^{2}$, MUHAMMAD TAYYAB RASHEED $^{3}$, MOHAMMED HABIB $^{4}$, NASEEM UL HAQ ${ }^{5}$, \\ SAKHAWAT KHAN 6 \\ ${ }^{1}$ Associate Professor ENT, Azra Nahid Medical College, Lahore \\ ${ }^{2}$ Senior Registrar, Chaudhary Muhammad Akram Teaching and Research Hospital/ Azra Nahid Medical College, Lahore \\ ${ }^{3}$ Assistant Professor ENT Aziz Bhatti Shaheed Teaching Hospital, Nawaz Sharif Medical College Gujrat \\ ${ }^{4}$ Associate Professor ENT Peshawar Medical College \\ ${ }^{5}$ Assistant Professor ENT department Kuwait Teaching Hospital/ Peshawar Medical College, Peshawar \\ ${ }^{6}$ Senior Registrar Department of ENT Khyber Teaching Hospital, Peshawar \\ Corresponding author: Dr. Rehan Saleem, E-mail: rehan.saleem85@gmail.com, Contact: +923217745772
}

\begin{abstract}
Objectives: To study treatment outcome of positional vertigo in adult patients of 15-40 years of age.

Place and duration of study: This study was conducted at ENT department of Chaudhary Muhammad Akram Teaching \& Research Hospital, Lahore and Kuwait Teaching Hospital, Peshawar from June 2019 to June 2020.

Material and Methods: 50 patients with diagnosis of benign positional vertigo were included in the study. Patients were treated and their outcomes were analyzed.

Results: 50 patients of age between 15-40 years were included in the study. The analysis of treatment outcome showed that 35 patients $(70 \%)$ showed maximum improvement in vertigo with medical treatment, 10 patients $(20 \%)$ showed improvement in their symptoms with positional exercises and 5 patients (10\%) showed improvement by combined treatment.

Conclusion: Benign paroxysmal positional vertigo (BPPV) is the most common peripheral vestibular disorder and presents as brief, episodic, positional provoked vertigo. The diagnosis can be made through clinical history along with diagnostic maneuvers and it can have a considerable impact on the quality of life. The medical treatment with vestibular dilators and vestibular suppressant is best option, with particle repositioning maneuver (Epley maneuver) for resistant and recurrent patients.
\end{abstract}

Key words: Vestibular exercises, Dizziness, Positional vertigo, Dis equilibrium.

\section{INTRODUCTION}

The symptoms of benign paroxysmal positional vertigo (BPPV) include a sudden and transient sensation of spinning, as well as distinct nystagmus. The symptoms are created by head movements driven by gravity and range from mild episodes to dizziness, which can cause nausea or vomiting and severely interfere with daily activities.

BPPV is the most prevalent cause of dizziness because the signal deviation from the semicircular canal can generate the illusion of movement $(1,2)$. BPPV can occur at any age, but the maximum occurrence occurs between the ages of 50 and 60 . In adults it occurs in 18$25 \%$, but disproportionately affects women in a ratio of 2 or 3 to 1 . No gender differences were observed in young patients $(3,4)$.

The high incidence of BPPV and several months delay in diagnosis and treatment are not uncommon. In a cross-sectional examination of 100 senior patients, it was discovered that $9 \%$ of those with symptoms unrelated to balance issue had never heard of BPPV (5).

The canalithiasis and cupulolithiasis models are the two most popular explanations for BPPV pathogenesis.

The particulate matter adheres to the cupula itself in the cupulolithiasis model. As a result of this cupular loading, the system becomes sensitive to gravitational forces, and the resulting variations in cupular deflection create anomalous motion impressions (6).

Free-floating particles within the canal lumen are cited as the cause of canalithiasis in the canalithiasis model.
Saccular otoconia are less likely to be engaged due to their distance from the semicircular canals.

The majority of BPPV instances are idiopathic in nature and are caused by macular degeneration. Secondary causes include otologic and non-otologic operations, head trauma, and any other means by which a significant mechanical force reaches the inner ear $(7,8,9)$ Other causes include vestibular neuritis, Meniere's illness, and sudden sensorineural hearing loss (SNHL) $(10,11,12)$.

BPPV can affect any of the three semicircular tubes, although the posterior tubular deformity is the most prevalent (80-90 percent) and is the region of the vestibular labyrinth that is most affected by gravity (8). The canal's uncut edge is the only spot where debris can enter or escape. Due to the tube's superb orientation, each tube particle rises solely through natural head motions, which are only $10 \%$ to $20 \%$ and $3 \%$ of the time anyway $(9,13,14)$.

People with BPPV often feel that the environment is circulating in the sound field. These dizzy spells normally last less than 20 seconds (although they can continue longer for horizontal tubes), however they can be accompanied by an imbalance. Furthermore, BPPV subversive symptoms develop suddenly and are provoked by certain head movements (such as neck extension, forward bending or rollover in bed) $(9,15)$. The position of the back can usually predict the injured ear $(16,17,18)$. There are patients with atypical medical history. This is why we check out Dix Halpike and the horizontal channels in every dizziness. 
Most BPPVs are idiopathic, but possible secondary causes include early trauma, vestibular neuritis, migraine or previous surgery. Physicians should be aware that BPPVlike causes of the central nervous system can signify more serious illnesses that require immediate treatment $(19,20$, $21,22)$.

The Dix-Hallpike operation is the certified test of the BPPV posterior canal. This causes proper nystagmus. Late start, short duration, torsional direction and pleasure, reversibility, and tiredness are all characteristics of nystagmus. The sensitivity and specificity of procedures performed in the office range from 79 to 82 percent and 71 to 75 percent, respectively $(23,24)$. Patients with problems in the neck, back, abdomen and buttocks may need special attention during the diagnostic procedure, so caution should be exercised (25).

Horizontal BPPV is diagnosed with a supine roll test, often known as the Pagnini McClure procedure (11). For the supine roll test, the patient is positioned supine in a neutral head position with their face pointed upwards. The head is then rapidly shifted 90 degrees to one side, and the eyes are checked for bidirectional and horizontal nystagmus.

The BPPV of the upper tube is relatively small (18). Anatomically, the upper semicircular tube and the opposite opposing posterior tube are in the same plane (27). Therefore, the manipulation of Dix-Hallpike is a test of these two objects. The torsion component of the positive Dix-Hallpike test of the BPPV root canal is the same as the posterior ear canal, with the vertical component of the downward pulse being the only difference (27).

Overall, BPPV can be diagnosed based on clinical history and distinctive nystagmus findings during provocative manoeuvres. Additional techniques, such as advanced electrical vestibular and auditory tests, are usually not necessary to identify isolated BPPV.

Patients should be informed of BPPV's favorable prognosis, which includes about $25 \%$ of patients experiencing spontaneous remission within one month and up to $50 \%$ of patients experiencing spontaneous remission after three months (28). While observation is an option, adequate treatment can help minimize the discomfort and potential danger caused by episodic vertigo (6). Furthermore, both treated and untreated patients can experience unpredictable relapses and remissions (4). As a result, education aids patients in anticipating and dealing with recurrences. More outdated treatments, such as severe inpatient positioning exercises and vestibular suppressant medications, have shown promising outcomes as simple, effective in-office therapies as our understanding of BPPV has evolved (29).

\section{MATERIAL AND METHODS}

The study was conducted in the department of ENT Chaudhary Muhammad Akram Teaching \& Research Hospital, Lahore and Kuwait Teaching Hospital, Peshawar from June 2019 to June 2020. Fifty patients were included in the study.

Inclusion criteria: Patients between 15-40 years of age, belonging to either sex, with history of vertigo, dizziness and unsteadiness symptoms due to positional changes were included in the study
Exclusion criteria: All the patients with some vertebral disease and systemic disease were excluded from the study.

Positional vertigo patients coming to hospital were studied regarding etiology of disease and treatment outcome and results of study were analyzed.

\section{RESULTS}

Among a total of 50 patients, 20 patients (40\%) were between 15-25 years, 20 patients (40\%) were between 2550 years and 10 patients (20\%) were between $50-60$ years as shown in Table-1.

Table 1. Age distribution:
\begin{tabular}{|l|l|l|}
\hline No of patients & Age (years) & Percentage $(\%)$ \\
\hline 20 & $15-25$ & $40 \%$ \\
\hline 20 & $26-35$ & $40 \%$ \\
\hline 10 & $36-40$ & $20 \%$ \\
\hline
\end{tabular}

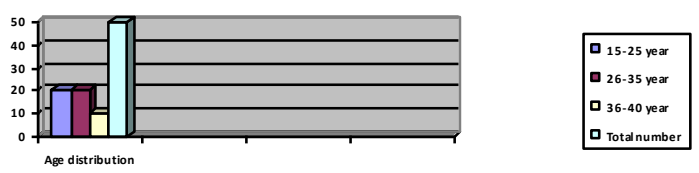

On analysis of sex distribution 15 patients (30\%) were male and 35 patients (70\%) were female as shown in Table-2.

Table 2. Sex distribution:

\begin{tabular}{|l|l|l|}
\hline Sex & No of patients & Percentage (\%) \\
\hline Male & 15 & $30 \%$ \\
\hline Female & 35 & $70 \%$ \\
\hline
\end{tabular}

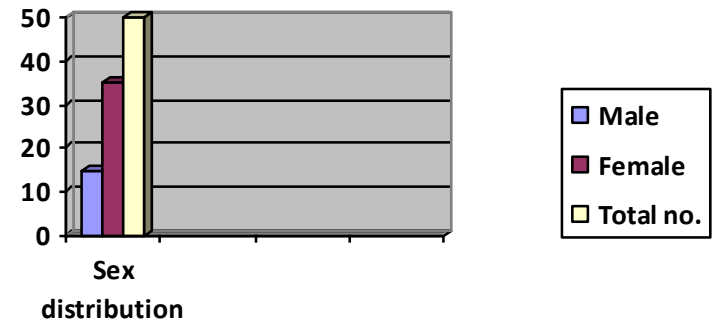

The analysis of treatment outcome revealed that 35 patients $(70 \%)$ showed maximum improvement in vertigo with medical treatment, 10 patients (20\%) showed improvement in their symptoms with positional exercises while 5 patients $(10 \%)$ were improved by combined treatment as shown in Table-3.

Table 3. Treatment method:

\begin{tabular}{|l|l|l|}
\hline Treatment method & $\begin{array}{l}\text { Number of } \\
\text { patients }\end{array}$ & Percentage (\%) \\
\hline Medical treatment & 35 & $70 \%$ \\
\hline Positional exercises & 10 & $20 \%$ \\
\hline Combined treatment & 5 & $10 \%$ \\
\hline Total & 50 & $100 \%$ \\
\hline
\end{tabular}




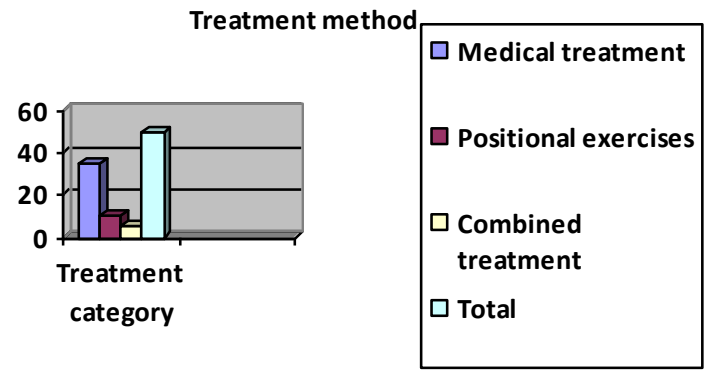

\section{DISCUSSION}

In our study, 50 patients with vertigo were included. Amongst these, 15 patients were male while 35 were female showing a female preponderance. 20 patients were between the ages of $15-25$ years and 20 patients were between 26-35 years while 10 patients were from 36-40 years of age. Age range was from 15year to 40 years.

Our study's demographic data was similar to that of prior studies, with a female majority of $62.1: 37.9 \%$.

Depending on the channel involved, the healing rate of BPPV is high, regardless of whether the relocation operation was performed correctly or not. Responses to treatment after single treatment ranged from $37 \%$ to $87 \%$. There were no statistically significant relationships between age, gender, cast type, symptom duration, and the number of relocation acts required to relieve symptoms.

BPPV is reported to include non-single PSC sites, and patients with BPCV LSC and multiple root canals may require more treatment visits (1).

2-4 also reported that homogeneous LSC BPPV treatment outcomes were worse than those of LSC BPPV at ground temperature. In our study, 35 patients received medical treatment, 10 LSC BPCV patients received treatment after one or two relocation surgeries, and five patients had to receive concomitant treatment.

Patients who received advanced BPPV treatment or experienced traumatic BPPV had lower cure rates, but there was no relationship between the duration of the symptom and the number of treatments in the study. We also found no difference in the number of treatments in patients with a history of BPPV lasting less than one month or more than one month.

Our study shows that people with high blood pressure need more visits compared to people with normal blood pressure. Osteoporosis, manieor disease, and central nervous system pathology have all been linked to BPPV recurrence in earlier investigations $(18,19,20)$. The presence of comorbidities such as hypertension and diabetes greatly raises the chance of recurrence, while hypertension greatly increases the number of recurrences, according to the authors. According to a study by Shim et al., the incidence of hypertension was $15 \%$. (21).

In a previous study by Shoman et al. (22) \& Halker et al. (23) As can be seen in Figure, the incidence of migraines in BPPV patients was significantly higher compared to the control group. The link between migraines and BPPV is unclear. It has been noted that migraine can cause spasm in the labyrinth artery, cause ischemia and promote spontaneous conical release from its conical macula (2). In 1956, Lindsay and Hymenway identified a blockage of the equilibrium artery in BPPV patients after vestibular neuritis. They showed an upper balance ganglion and a well-managed ear dissolver (24). In 2003, Before death, Gachek investigated the temporal bones of five patients with a history of BPPV and discovered that degeneration of the vestibular nerve cells is more prevalent than pathology of the sense organs. The authors have shown that there are temporary bones in only a few cupshaped sediments (25). Arterial hypertension was the most common pathology in a retrospective analysis by Farialli et al., with a frequency of 35.6 percent, followed by hypercholesterolemia, hyperglycemia, ischemic heart disease, cerebrovascular illness, and patients with proximal dizziness. They found that patients with multiple vascular factors needed significantly more surgeries than patients with or without vascular factors alone. 26 In previous studies and findings, it is believed that complex hypertension may promote vestibular dysfunction. Ischemia causing macular degeneration and detachment from the ear.

\section{CONCLUSION}

The most common peripheral vestibular condition is benign paroxysmal positional vertigo, which manifests as short, episodic, positional-induced vertigo. Clinical history and diagnostic techniques are usually enough to make the diagnosis, and no more testing is usually required. It has a significant impact on quality of life, although being benign and typically self-limiting. As a result, regular medical therapy is essential, with or without positioning exercises. Fortunately, a better knowledge of the underlying pathophysiology has led to the development of a number of repositioning procedures to aid clinicians in the treatment of this prevalent ailment. The posterior canal closure procedure, which is both safe and effective, can help a small number of patients with intractable BPPV.

\section{REFRENCES}

1. Hanley K, O'Dowd T, Considine N. A systematic review of vertigo in primary care. $\mathrm{Br} \mathrm{J}$ Gen Pract 2001;51(469):666671.

2. Katsarkas A. Benign paroxysmal positional vertigo (BPPV): idiopathic versus post-traumatic. Acta Otolaryngol 1999;119(7):745-749.

3. von Brevern M, Radtke A, Lezius F, et al. Epidemiology of benign paroxysmal positional vertigo: a population based study. J Neurol Neurosurg Psychiatry 2007;78(7):710-715.

4. Kim J-S, Zee DS. Benign Paroxysmal Positional Vertigo. N Engl J Med 2014;370(12):1138-1147.

5. Mizukoshi K, Watanabe Y, Shojaku H, Okubo J, Watanabe I. Epidemiological studies on benign paroxysmal positional vertigo in Japan. Acta Otolaryngol Suppl 1988;447:67-72.

6. Bhattacharyya N, Gubbels SP, Schwartz SR, et al. Clinical practice guideline: benign paroxysmal positional vertigo (update). Otolaryngol Head Neck Surg 2017;156(Suppl 3):S1-S47.

7. Fife D, FitzGerald JE. Do patients with benign paroxysmal positional vertigo receive prompt treatment? Analysis of waiting times and human and financial costs associated with current practice. Int J Audiol 2005;44(1):50-57.

8. Oghalai JS, Manolidis S, Barth JL, Stewart MG, Jenkins HA. Unrecognized benign paroxysmal positional vertigo in elderly patients. Otolaryngol Head Neck Surg 2000;122(5):630-634. 
9. Parnes LS, Agrawal SK, Atlas J. Diagnosis and management of benign paroxysmal positional vertigo (BPPV). CMAJ 2003;169(7):681-693.

10. Parnes LS, McClure JA. Free-floating endolymph particles: a new operative finding during posterior semicircular canal occlusion. 1992. Laryngoscope 2015;125(5):1033.

11. Lee S-H, Kim JS. Benign paroxysmal positional vertigo. J Clin Neurol 2010;6(2):51-63.

12. Kao WTK, Parnes LS, Chole RA. Otoconia and otolithic membrane fragments within the posterior semicircular canal in benign paroxysmal positional vertigo. Laryngoscope 2016;90:709-714.

13. Kansu L, Aydin E, Gulsahi K. Benign paroxysmal positional vertigo after nonotologic surgery: case series. J Maxillofac Oral Surg 2015;14(Suppl 1):113-115.

14. Park S-K, Kim SY, Han K-H, Hong SK, Kim JS, Koo $\mathrm{J}-\mathrm{W}$. Benign paroxysmal positional vertigo after surgical drilling of the temporal bone. Otol Neurotol 2013;34(8):14481455.

15. Lee $\mathrm{N}-\mathrm{H}$, Ban J-H, Lee K-C, Kim SM. Benign paroxysmal positional vertigo secondary to inner ear disease. Otolaryngol Head Neck Surg 2010;143(3):413-417.

16. Balatsouras DG, Ganelis P, Aspris A, Economou NC, Moukos A, Koukoutsis G. Benign paroxysmal positional vertigo associated with Meniere's disease: epidemiological, pathophysiologic, clinical, and therapeutic aspects. Ann Otol Rhinol Laryngol 2012;121(10):682-688.

17. El-Saied S, Joshua B-Z, Segal N, Kraus M, Kaplan DM. Sudden hearing loss with simultaneous posterior semicircular canal BPPV: Possible etiology and clinical implications. Am J Otolaryngol 2014;35(2):180-185.
18. Anagnostou E, Kouzi I, Spengos K. Diagnosis and treatment of anterior-canal benign paroxysmal positional vertigo: a systematic review. J Clin Neurol 2015;11(3):262-267.

19. Hornibrook J. Benign Paroxysmal Positional Vertigo (BPPV) history, pathophysiology, office treatment and future directions. Int J Otolaryngol 2011;2011:835671.

20. Dix MR, Hallpike CS. The pathology symptomatology and diagnosis of certain common disorders of the vestibular system. Proc R Soc Med 1952;45(6):341-354.

21. Shim DB, Ko KM, Kim JH, Lee W-S, Song MH. Can the affected semicircular canal be predicted by the initial provoking position in benign paroxysmal positional vertigo? Laryngoscope 2013;123(9):2259-2263.

22. Shoman N, Longridge N. Cerebellar vermis lesions and tumours of the fourth ventricle in patients with positional and positioning vertigo and nystagmus. J Laryngol Otol 2007;121(2):166-169.

23. Halker RB, Barrs DM, Wellik KE, Wingerchuk DM, Demaerschalk BM. Establishing a diagnosis of benign paroxysmal positional vertigo through the dix-hallpike and side-lying maneuvers: a critically appraised topic. Neurologist 2008;14(3):201-204.

24. López-Escámez JA, López-Nevot A, Gámiz MJ, et al. [Diagnosis of common causes of vertigo using a structured clinical history]. Acta Otorrinolaringol Esp 2000;51(1):25-30.

25. Humphriss RL, Baguley DM, Sparkes V, Peerman SE, Moffat DA. Contraindications to the Dix-Hallpike manoeuvre: a multidisciplinary review. Int J Audiol 2003;42(3):166-173.

26. Hornibrook J. Horizontal canal benign positional vertigo. Ann Otol Rhinol Laryngol 2004;113(9):721-725. 